\title{
How Space Experimentation Can Inform Design: Immersive Virtual Reality as a Design Tool
}

\author{
Ana Moural \\ ISCTE-IUL, Portugal \\ ana_margarida_moural@iscte.pt \\ Sara Eloy \\ ISCTE-IUL / ADETTI-IUL, Portugal \\ sara.eloy@iscte.pt
}

\author{
Miguel Sales Dias \\ Portugal \\ miguel.dias@microsoft.com \\ Tiago Pedro \\ ISCTE-IUL, Portugal \\ tiago.miguel.pedro@gmail.com
}

Microsoft Language Development Center / ADETTI-IUL,

\begin{abstract}
Immersive virtual reality allows us to experience the space even before it's constructed. In this way, the use of such an environment plays a decisive role in the design process in architecture. The spaces that architects design may now be evaluated in a real scale mode which enable the understanding of problems and/or opportunities that space bring to the buildings. The goal of this paper is to define the methodological framework for using an immersive virtual environment, namely CaveH, in an Architectural design process by exploring the use of real-time rendering. We intend that the developed software and infrastructure will be used in a very easy way as a valid design tool to any professional. Two 3d models were experiment along this research each one of them regarding to a different type of design problem.
\end{abstract}

Keywords: Immersive virtual reality; Design; 3D modelling; Cave; Experience.

\section{Introduction}

In a moment that increasingly complex geometries are arising in architecture, immersive virtual reality emerges as a powerful tool to architectural design.

"Whereas real space is limited to reality, virtual [lat.: existent due to power or possibility] space is created by human mind and thus only limited by thoughts. This freedom gives virtual space unlimited qualities like free space-directions, fusion of object and space, free shapes, time and movement as changing factor, zero gravitation, free definable forces, etc.." (Kieferle, 2000)

The use of 3D digital models in Architecture enable by the constant advances in computer graphics is becoming increasing accurate. Virtual models seen in laptops lack the real scale of the building which is a disadvantage to real experimentation of space. This paper explores the use of virtual immersive environments (VIE) - systems which enables the visualization of environments (outdoor, indoor, etc) as well as the navigation and interaction with a real time environment - in architecture design processes.

In fact, VIE presents an alternative system to see architectural spaces, providing the possibility of testing architecture models at real scale and in a virtual environment. The software we are using - CaveH - is open to many possibilities of exploration since it has been developed by ADETTI-IUL (Centre for Research in Information Systems and Advanced Technologies, University Institute of Lisbon, Portugal) team so it is possible to do constant tweaks and improvements through a tight cooperation between architecture and engineering. The 3D model is designed through a process which tends to become increasingly user friendly with the enormous advantage of being a much cheaper approach because it can reuse the 3D models already produced, independently of physical model production. The real-time rendering is a fundamental issue, i.e., giving the possibility to roam freely through the space in real time. The presented research in architecture follows several works developed by ADETTI's team, like CAVE HollowSpace present in Centro de Ciência Viva do Lousal, Portugal (Costa et al, 2010).

Several architecture faculties and research centers around the world are using VIE to help the simulations and evaluation of space. In LivingLab CASALA (Centre for Affective Solutions for Ambient Living Awareness, Ireland, http://www.casala.ie/casalaliving-lab.html) specific environments for an integrated monitoring of citizens with reduced mobility are tested. Also, the $A \& M$ University (Texas, USA) and the Art and Technology Studios VRLab (Chicago, USA) are two examples of universities that hold virtual reality systems that allow real-time navigation using commercial software (Autodesk Navisworks and Autodesk 3ds Max). Although the process of modeling and navigation is facilitated by relying on standard software, there are some limitations, especially in terms of the manipulation of the physical properties of objects and the customization of the interaction, which makes the experience less immersive and the exploration of different scenarios more limited. 
Based on these two references, it is possible to emphasize the importance these environments have within the architectural design process and how import a user friendly platform is.

In Working Development subsection, we present the physical characteristics of our CAVE structure, some theoretical assumptions taken and the choices made in the course of software evolution. We finish weighing the advances that have been made and how the results lead to the possibility of applying the software in different situations than those in which it has been tested, finally we present some of the goals that we hope to achieve in the short / medium term.

\section{Working Development}

One of the goals of this work was to ensure that we have a customizable platform according to the needs of the 3D models and the projects to be developed. CaveH Spwaner, developed in ADETTI-IUL eased this requirement, although it had already been developed for other purposes. This platform, developed essentially to comply with video games requirements, was developed with many limitations regarding the needs of architectural design.

The intention of using the platform to manipulate an architectural environment required numerous adjustments to the software. CaveH Spawner was then challenged to be more than a navigation tool that mainly served the purpose of navigation in a virtual environment modeled for the recreation of a real space existing. The purpose was indeed to adapt it to go from the simple navigation tool to manipulation / experimentation tool that will serve the architectural design work.

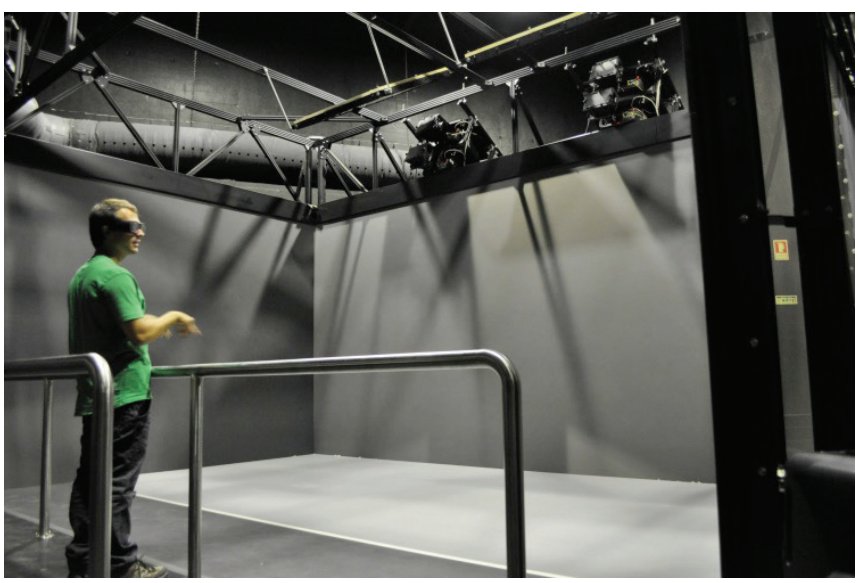

Figure 1: CAVE facility in Centro de Ciência Viva do Lousal

\section{Infrastructures}

The Centro de Ciência Viva do Lousal currently uses a CAVE (Figure 1) for thematic virtual tours to the regional mines that once existed in the village where the center is located. The mines were partially modeled in $3 \mathrm{D}$ in order to be presented to the public and especially to create a more technological and interactive experience of what used to be an important impetus to the region.
This CAVE facility has overall dimensions of $5.60 \times 2.70 \times 3.40 \mathrm{~m}$ and comprises six projection planes (one left, one right, two front and two on the ground), each targeted by two projectors. However, only the frontal and horizontal allow stereo display through the use of polarized glasses, the remaining supplement the peripheral vision. This system also has a sound system installed which promotes a greater user integration in the simulated environment, as well as the stimulation of other senses than just the visual (Bastos \& Dias, 2008).

Following what was done in the Lousal Center, later in ADETTI-IUL a smaller structure called PocketCAVE was installed. This smaller CAVE have the main goal of approaching this tool to the scientific community of ISCTE-IUL and to all exterior stakeholders in order to provide an infrastructure dedicated to research and practice. This infrastructure has one projection screen of $3.29 \times 2.10 \mathrm{~m}$ running with a single projector and it's a portable CAVE that can be easily transported and assembled in any location. Although the projection screen enhances the experience exponentially, the projection can be done over any smooth surface which means that the maximum portability will only depend on the transportation of the projector. In the PocketCAVE we consider the experience only to be semi-immersive due to the dimensions of space as well as the projection area. The navigation in the virtual environment is currently being made possible through the use of keyboard + mouse or pair WiiMote + Nunchuck controllers (Nintendo Wii).

\section{Software}

The software CaveH Spawner is currently used in this two centers (ADETTI-IUL and Centro de Ciência Viva do Lousal) with very different physical structures and was developed by a team that began its work primarily from the facilities of the Centro de Ciência Viva do Lousal (Costa et al, 2010). This software is not entirely dependent on standard software but instead it is based on a conjunction of custom in-house software and open software. Blender is the $3 \mathrm{~d}$ modeling open-source software chosen to make the transition from the 3D model (done in any 3d software) and CaveH Spawner, which is where the immersive environment is launched. Blender is used not only as an easy that enable the enable to convert from many commercial products file types to the CaveH format, and more important, it is used as an authoring tool. In Blender there are many different steps to do: all the models became entities and user can set his own characteristics like physics properties, interaction, etc. This is the most important difference between this work and others, in the end it will serve the purpose of having a system where we can test scenarios in an easily and cheaper way.

In Blender software each model must have a physical property in order to be corrected modeled in the VR environment, this process will transform models in entities. 


\section{Entities}

Since the beginning of the work in Blender, any object present in the scene has been set to be one of the following entities:

- Misc model (object represented in the physics system): static objects from the scene with which the user cannot interact. These entities will create "bounding boxes" (visible in the physical system but invisible in the navigation space) that prevent approximation to objects and consequently the interaction. Therefore there are objects of low relevance in the scene;

- Map geometry (object represented in the physics system): This should be the entity to assign to all objects with which one can interact. It applies, for instance, to a wall where there are gaps or any other type of elements defining a path;

- Info player start (element that defines the starting location of the route): defines the starting location of the character in virtual space;

- Object without physical representation in the system: entity still under development and testing that aims to enable, when paired with one of the first two mentioned above, the overlap with another object in wireframe mode. This option aims to create an alternative view that combines solid volumes with the polygons that define the respective surfaces of the object.

\section{Avatar dimensions}

The user is represented by an object that is defined in the scene as a camera. The user is represented in the physics engine as a capsule where you can change the default parameters regarding height or diameter of the avatar. Thus the user can fit the scale of the virtual environment quite accurately since the change is made using numerical parameters.

\section{Avatar movement velocity}

The avatar speed, both vertical and horizontal, is also fully customizable using numeric parameters. This is a major feature in the definition of the model before exporting to CaveH Spawner that is fundamental to the perception of the space especially in indoor environments. However there are some possible drawbacks, the immersive experience together with a high speed movement can take to an incorrect perception of space, as well as the possible motion nausea of the user. So, it is highly important to configure the avatar speed and its size carefully.

\section{Distance triggers}

With the objective of increasing the user interaction with the scene, it is still possible to add actions triggered by proximity to a particular object. You can assign this feature to any element of the scene and also set its visibility - the entrance in the area defined as activator will trigger the action, even if the object is invisible. There are currently available some triggers:
- Change map (it will trigger a change in the level/map to another one)

- Animation start (it will start an animation, that can be, for instance, a door opening)

- Movie play (It can be used to start a video during the virtual roaming. This way it could illustrate some project aspects that are important to be told or visualized in a video)

- Sound play (It starts a sound. It can be used to simulate crowded spaces or noise)

- Camera shake (It shakes the camera, it can be used to

- simulate an earthquake or the simple movement of a person in an elevator)

There are three more triggers, that were not found any utility in this context, but they can still be used in other type of scenarios:

- Add object (When the user enters the trigger area, a new object is added to the scene)

- Change gadget (The user can carry objects called gadgets, there are a trigger that can give new gadgets to the user)

\section{Case Study and results}

We tested systematically different models: in order to assess the benefits of virtual reality in the context of architectural design a partial model of an area of the city of Lisbon to where a design project was being developed was used; a model of our University ISCTE-IUL campus was done to perform an evaluation of the space usability. Through these two examples we confirm that platform still does not always have the expected solutions to the issues raised by architectural models. In this sense it is possible to see that the platform still have to be improved for this type of use.

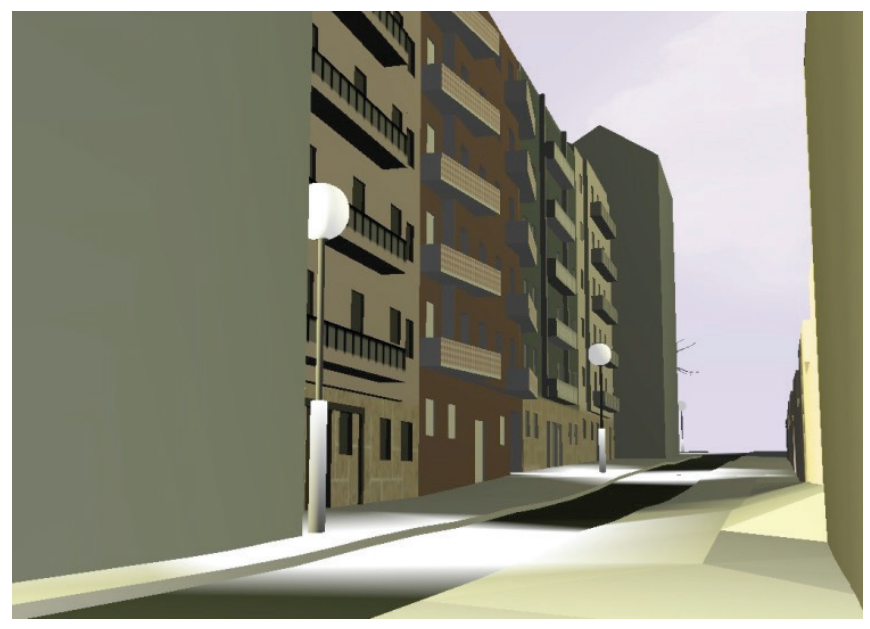

Figure 2: CaveH screenshot. Real time navigation in the partial model of an area of the city of Lisbon

Both Blender and CaveH Spawner interfaces have too much information and work possibilities. However, it turns out that Blender is still a barrier to a greater recognition of the CAVE system by the community of architects and students of 
architecture. The possibility of adapting the CaveH Spawner software in order to use a common architectural software would allow a more active dialogue with students and architects. Blender is a dedicated software for modeling, animation and 3D models rendering, but it's not well suited for using with the type of CAD modeling taken by architects and hardly can be used as the single tool in the architecture design process.

Blender was customized by ADETTI-IUL engineers in order to support the entities' model, which makes this work so useful. To became more friendly to the architecture practice we are researching on the hypothesis of turning Blender tasks into automatic tasks or in a guided/assisted way, using a tutorial or a wizard.

The usefulness of this platform as a tool for architecture is independent of concern about the absolute realism of what is presented since the constant shift between representation and visualization enable the feedback we had from pedestrians.

"Hardly anything in VR is like in reality, however VR can be used to project a certain part of reality. (...) Similar to the mental "concept", most properties of the things can't be perceived visually in reality. VR offers a chance to develop a visual representation of properties." (Kieferle and Wossner, 2001)

To answer to this challenging we have started to develop a way to display wireframe objects. The goal of this new invention is to enable a more automatic and inside the design process so that, with an investment in the modeling of the space and textures mapping and its materials, it is possible to test an architectural space quite embryonic, only from the point of view of experimentation, while space is still the result from the combination of planes, surfaces and volumes.

\section{Conclusions and Future Work}

Our work has been developed with researchers from two distinct areas, architecture and computer engineering, that daily are working together, testing and interacting in order to make the software advance towards a better solution for all the challenges proposed by the different stakeholders.

Within the architecture goals, there is a set of elements that we will try to achieve in the sort-term. In fact, there are other elements, besides the model itself, that contribute strongly to the space perception and that can change the way the users uses the virtual environment while his navigation.

We found that it would be important to have features like the possibility of simulate the day-night cycle, in order to have a viable illumination model, dependent of the hour of the day, one of the most important feature in architectural proceed. As well as have a way to simulate the loudness of the environment, depending on the location, the time of the day, as well as the surroundings, giving the possibility to the user, even without seeing, having the perfect notion of the space and time, through the sound.

We also plan to create a system where the users can simulate thermal variations during the navigation. Also, providing stimulus to the other senses, as providing smells, simulate winds or producing heat (as seen in the work developed by Azevedo, 2013), is a way to go in the future. It is known, that the vision is the most active sense in this experience, but when experienced with stimulus to other senses, it loses some importance (Azevedo, 2013), changing the way how the user will perceive and roam the virtual space.

Finally, it was proposed as a crucial tool the inclusion of the interaction using the Microsoft Kinect sensor. That would create a seamless navigation, using just the user's body, as well as the possibility to interact with the space with the user's body. It would also open the possibility to the system to be used by people with some kind of disabilities.

The intentions for future work are all planned and feasible in the mid-term within this open platform. This input given by us to the engineering team, is only possible because we have a straight collaboration that give us the possibility of influence the development cycle, with new kinds of interactions and customizations. In the end, this opens endless opportunities to the CAVE when compared with other "standard" systems that are tight connected to the closed software on which they are based.

\section{Acknowledgment}

This paper has been partially supported by the QREN 7943 CNG Contents for Next Generation Networks project which is co-funded by Microsoft, the Portuguese Government, and the European Structural Funds for Portugal (FEDER), through COMPETE and QREN.

\section{References}

Azevedo, A. S. (2013). 3-D Sound Enhanced Presence in Virtual Environments. s.I., s.n.

Bastos, P., Dias, M. S., 2008. Experiência de realidade virtual ilmersiva no ambiente CaveHollowspace do Lousal [3rd Human-Computer Interaction Conference Proceedings] Évora, Portugal

Costa, V. C.; Dias, J. M. S.; Pereira J. M. (2010): "Tecnologias CAVEHOLLOWSPACE para a Mina do Lousal"; Instituto Superior Técnico, Lisbon, Portugal

Kieferle, J., Wossner, U., 2001. Showing the invisible: Seven rules for a new approach of using immersive virtual reality in architecture. Process [19th eCAADe Conference Proceedings] Helsinki, 29-31 August 2001, pp. 376-381

Kieferle, J. B. (2000): "Virtual space - new tasks for architects"; Universitat Stuttgart; Germany. 